\title{
MicroRNA expression profiles associated with pancreatic adenocarcinoma and ampullary adenocarcinoma
}

\author{
Nicolai A Schultz ${ }^{1,2}$, Jens Werner ${ }^{3}$, Hanni Willenbrock ${ }^{4}$, Anne Roslind ${ }^{5}$, Nathalia Giese ${ }^{3}$, \\ Thomas Horn ${ }^{5}$, Morten Wøjdemann² and Julia S Johansen ${ }^{6,7}$ \\ ${ }^{1}$ Department of Surgical Gastroenterology and Transplantation, Rigshospitalet, Copenhagen University \\ Hospital, Copenhagen, Denmark; ${ }^{2}$ Division of Surgery, Department of Gastroenterology, Herlev Hospital, \\ Copenhagen University Hospital, Copenhagen, Denmark; ${ }^{3}$ Department of General, Visceral, and Transplant \\ Surgery, University of Heidelberg, Heidelberg, Germany; ${ }^{4}$ Exiqon A/S, Vedbaek, Denmark; ${ }^{5}$ Department of \\ Pathology, Herlev Hospital, Copenhagen University Hospital, Copenhagen, Denmark; ${ }^{6}$ Department of \\ Oncology, Herlev Hospital, Copenhagen University Hospital, Copenhagen, Denmark and ${ }^{7}$ Department \\ of Medicine, Herlev Hospital, Copenhagen University Hospital, Copenhagen, Denmark
}

\begin{abstract}
MicroRNAs have potential as diagnostic cancer biomarkers. The aim of this study was (1) to define microRNA expression patterns in formalin-fixed parafin-embedded tissue from pancreatic ductal adenocarcinoma, ampullary adenocarcinoma, normal pancreas and chronic pancreatitis without using micro-dissection and (2) to discover new diagnostic microRNAs and combinations of microRNAs in cancer tissue. The expression of 664 microRNAs in tissue from 170 pancreatic adenocarcinomas and 107 ampullary adenocarcinomas were analyzed using a commercial microRNA assay. Results were compared with chronic pancreatitis, normal pancreas and duodenal adenocarcinoma. In all, 43 microRNAs had higher and 41 microRNAs reduced expression in pancreatic cancer compared with normal pancreas. In all, 32 microRNAs were differently expressed in pancreatic adenocarcinoma compared with chronic pancreatitis (17 higher; 15 reduced). Several of these microRNAs have not before been related to diagnosis of pancreatic cancer (eg, miR-492, miR-614, miR622). MiR-614, miR-492, miR-622, miR-135b* and miR-196 were most differently expressed. MicroRNA profiles of pancreatic and ampullary adenocarcinomas were correlated (0.990). MicroRNA expression profiles for pancreatic cancer described in the literature were consistent with our findings, and the microRNA profile for pancreatic adenocarcinoma (miR-196b-miR-217) was validated. We identified a more significant expression profile, the difference between miR-411 and miR-198 $\left(P=2.06 \times 10^{-54}\right)$ and a diagnostic LASSO classifier using 19 microRNAs (sensitivity 98.5\%; positive predictive value 97.8\%; accuracy $97.0 \%$ ). We also identified microRNA profiles to subclassify ampullary adenocarcinomas into pancreatobiliary or intestinal type. In conclusion, we found that combinations of two microRNAs could roughly separate neoplastic from nonneoplastic samples. A diagnostic 19 microRNA classifier was constructed which without micro-dissection could discriminate pancreatic and ampullary adenocarcinomas from chronic pancreatitis and normal pancreas with high sensitivity and accuracy. Ongoing prospective studies will evaluate if these microRNA profiles are useful on fine-needle biopsies for early diagnosis of pancreatic cancer.
\end{abstract}

Modern Pathology (2012) 25, 1609-1622; doi:10.1038/modpathol.2012.122; published online 10 August 2012

Keywords: ampullary adenocarcinoma; chronic pancreatitis; microRNA; normal pancreas; pancreatic adenocarcinoma; pancreatic cancer

Correspondence: Dr NA Schultz, MD, Department of Surgical Gastroenterology and Transplantation, C 2-12-2, Rigshospitalet, Blegdamsvej 9, Copenhagen DK-2100, Denmark.

E-mail: nicolai-schultz@dadlnet.dk

Received 8 August 2011; revised 12 April 2012; accepted 14 April 2012; published online 10 August 2012
Pancreatic cancer is the fourth most common cause of cancer death in United States and Europe. ${ }^{1,2}$ Less than $20 \%$ of the patients can be operated with curative intent and the 5-year survival after surgery is below $20 \% .^{3}$ Early diagnosis is difficult and the clinical and histological similarities between pancreatic cancer and chronic pancreatitis further complicate the differentiation between inflammation and cancer. 
Ductal adenocarcinomas are the most common pancreatic cancer. Twelve percent of all periampullary carcinomas are adenocarcinomas of the Ampulla of Vater (ampullary adenocarcinomas).,5 These patients have a better prognosis with 5-year survival after surgery of $>45 \% .^{4,5}$ One of the reasons is that even small ampullary adenocarcinomas cause jaundice and therefore more patients are operated at an early tumor stage and without lymph node metastasis. Furthermore, biological differences between pancreatic adenocarcinomas and ampullary adenocarcinomas exist. Ampullary adenocarcinomas are a heterogeneous group of pancreatobiliary adenocarcinomas deriving from the ductal epithelium and intestinal-like adenocarcinomas arising from intestinal mucosa of the papilla. Conflicting data have been reported about the frequency of these two histological types due to the absence of reliable and consistent histomorphological criteria for differential diagnosis. ${ }^{6-9}$

MicroRNAs are 19-25-nucleotide-long non-coding RNAs which after cleavage of a precursor into their mature form bind to the RNA-inducedsilencing-complex (RISC) and regulate gene expression posttranscriptionally by a binding of specific mRNA. MicroRNAs regulate many genes known to play important roles in oncogenesis, angiogenesis and tissue differentiation. ${ }^{10-15}$ In all, 1527 human microRNA sequences have been discovered to date and the number is increasing (miRBase. http:// www.mirbase.org, last accesses February 2012). A * in the name of a microRNA, for example, miR-148a* vs miR-148a, indicate microRNAs that originate from the same predicted precursor. MicroRNAs have highly tissue-specific expression patterns. ${ }^{15-19}$ In pancreatic adenocarcinomas, the expression of miR-15b, miR-21, miR-95, miR-103, miR-107, miR-148a, miR-155, miR-196a, miR-200, miR-210, miR-217, miR-221, miR-222 and miR-375 are different from tissue of normal pancreas and chronic pancreatitis. ${ }^{17,20-27}$ The results reported by Bloomston et $a l^{20}$ and Szafranska et $a l^{26}$ give promises of significant clinical impact of microRNA expression profiles to separate tissue from pancreatic adenocarcinomas from normal pancreas and chronic pancreatitis. The study by Szafranska et $a l^{26}$ included a combination of two microRNAs (the difference between miR-196a and miR-217) to separate pancreatic adenocarcinomas and chronic pancreatitis (ASURAGEN-test).

Previous studies have been performed on relatively small groups of patients and some are performed with the use of micro-dissection or other tumor cell enrichment. ${ }^{20,21,23,24}$ MicroRNAs are stable in formalin-fixed paraffin-embedded samples. ${ }^{28,29}$ Tumor cells in pancreatic ductal adenocarcinomas are often located in small groups surrounded by an abundant stromal tissue ${ }^{30}$ and information related to microRNAs from the stromal tissue may be lost if micro-dissection is applied.

The aim of this study was with the use of microRNA profiling to (1) define differences in microRNA expression patterns in formalin-fixed paraffin-embedded tissue from pancreatic adenocarcinomas, ampullary adenocarcinomas, normal pancreas and chronic pancreatitis without using micro-dissection and (2) discover new diagnostic microRNAs and combinations of microRNAs in pancreatic and ampullary adenocarcinoma tissue.

\section{Materials and methods}

\section{Patients}

In all, 277 patients operated with pancreatic resection at Herlev Hospital between 1976 and 2008. Included were only pancreatic adenocarcinomas of ductal origin $(n=170)$ and ampullary adenocarcinomas $(n=107)$ of both pancreatobiliary and intestinal type. Differentiation between pancreatic adenocarcinomas and ampullary adenocarcinoma subtypes was determined by a combination of gross pathology, microscopy and immunohistochemical analysis. In all, 257 patients underwent a pancreaticoduodenectomy (Whipple procedure), 13 a distal pancreatectomy and 7 a total pancreatectomy. An existing database with clinical information was updated March 2010. Characteristics of the patients are shown in Table 1.

\section{Controls}

Archival formalin-fixed paraffin-embedded tissue blocks from patients operated for chronic pancreatitis $(n=23)$ and of normal pancreas $(n=28)$ were

Table 1 Clinicopathological characteristics of the patients with pancreatic adenocarcinomas and ampullary adenocarcinomas

\begin{tabular}{lcc}
\hline Characteristic & $\begin{array}{c}\text { Pancreatic adenocarcinoma } \\
(\mathrm{n}=160)\end{array}$ & $\begin{array}{c}\text { Ampullary adenocarc. (pancreatobiliary type } \\
\mathrm{n}=48 ; \text { intestinal type } \mathrm{n}=48)\end{array}$ \\
\hline Age, years median (range) & $63(33-85)$ & $64(31-79)$ \\
Sex, male/female & $78 / 82$ & $44 / 52$ \\
Tumor stage, IA/IB/IIA/IIB & $9 / 11 / 41 / 99$ & $3 / 25 / 30 / 38$ \\
Histological grade undifferentiated/poor/moderate/well & $4 / 71 / 38 / 47$ & $12 / 26 / 26 / 32$
\end{tabular}

\footnotetext{
${ }^{\text {a }}$ Significant differences between groups.
} 
obtained from Department of General, Visceral and Transplant Surgery, University of Heidelberg, Department of Pathology at Herlev Hospital, Copenhagen University Hospital, and Department of Pathology at Haukeland University Hospital, Bergen. Normal pancreas tissue blocks were taken as a part of full organ procurement in organ donor patients and from patients with traumatic lesions in tissue around the pancreas that led to removal of healthy pancreas. Archival formalin-fixed paraffinembedded tissue blocks from patients operated for duodenal adenocarcinomas $(n=15)$ were obtained from Department of Pathology at Herlev Hospital, Copenhagen University Hospital and used in an attempt of subclassification of ampullary adenocarcinomas.

The study was approved by the local Ethical Committee (protocol H-KA-20060181) in Region Hovedstaden, Denmark. The use of control materials was approved by the Ethical Committees in Bergen, Norway and in Heidelberg, Germany.

\section{Immunohistochemical Analysis}

New sections from tumor tissue blocks from each patient were stained with hematoxylin and eosin and examined by two experienced pathologist (AR, TH). All tumors were classified and graded according to WHO Classification of Tumors. ${ }^{31}$ Differentiation between pancreatic and ampullary adenocarcinomas was determined by a combination of gross pathology and microscopy. The ampullary adenocarcinomas were subclassified into pancreatobiliary- or intestinal-type adenocarcinomas histologically and if necessary immunohistochemically by applying the following markers: cytokeratin 20, cytokeratin 7, CDX2, MUC1, MUC2 and MUC5.

\section{MicroRNA Analysis}

Three $10 \mu \mathrm{m}$ sections were cut from each of the formalin-fixed paraffin-embedded samples for RNA extraction and placed in a sterile Eppendorf tube. MicroRNAs were extracted using the High Pure miRNA Isolation Kit (Roche). In brief, the tissue sections were deparaffinized in xylene and ethanol, treated with proteinase K, finally RNA was isolated using the one-column spin column protocol for total RNA. The Concentration of RNA was assessed by absorbance spectrometry on NanoDrop X-1000 (Thermo Fisher Scientific, Inc.). The microRNA profiling was performed on TaqMan ${ }^{\circledR}$ Array Human MicroRNA A + B Cards v2.0 (Applied Biosystems) using the manufacture's reagents and instructions. Each array analyses 664 different human microRNAs and enables a comprehensive expression profile consistent with Sanger miRBase v14. Briefly, the RNA was transcribed into cDNA in two multiplex reactions each containing $200 \mathrm{ng}$ of RNA and either Megaplex RT Primer A Pool or Pool B pool using the TaqMan MicroRNA Reverse Transcription Kit in a total volume of $14 \mu \mathrm{l}$. Prior to loading, the 12 cycle preamplification reaction was performed using $2.5 \mu \mathrm{l}$ cDNA in a $25-\mu \mathrm{l}$ reaction. Each of the arrays was loaded with $800 \mu \mathrm{l}$ Universal PCR MasterMix assay containing $1 / 40$ of the preamplification reaction and run on the $7900 \mathrm{HT}$ Fast RealTime PCR System.

\section{Statistical Analysis}

Raw $C_{\mathrm{t}}$-values where pre-processed in the following steps: (1) missing values and $C_{\mathrm{t}}$-values above 32 was flagged; (2) repeat measurements (excluding flagged values) were averaged; (3) features that were flagged in more than a given percent of samples were removed from the data set; (4) missing values were set to $C_{\mathrm{t}}=40$; (5) quantile normalization was performed. ${ }^{32}$ For quality control of samples, the threshold in step 3 was set to $80 \%$. Normalized data were inspected for outliers and potential technical bias from sample quality, purification date and card batch. No heavy technical bias was observed. Twenty-one samples were identified as outliers. Most samples' $C_{t}$-density curves were bimodal with peaks around 29 and 40 . In some cases, the peak around 40 was relatively high compared with the peak around 29 and these samples corresponded well to outliers identified by principal component analysis. We therefore removed samples from the data set if the ratio between the peaks at $C_{\mathrm{t}}>32 \mathrm{vs}$ $C_{\mathrm{t}}<32$ was above 0.9 (outlier criteria 1: density ratio $>0.9$ ) or if their average correlation (Pearson correlation) with other samples in the data set was below 0.70 (outlier criteria 2: average correlation $<0.7$ ). Samples that were close to failing both criteria were also categorized as outliers (outlier criteria 3: density ratio $>0.8$ and average correlation $<0.77$ ). Samples that passed quality control was pre-processed as described above with the threshold in step 3 now set to $95 \%$. Analyses comparing $\Delta C_{t}$ of two individual miRNAs between samples are based on un-normalized $C_{\mathrm{t}}$-values while the remaining analyses are based on normalized data. Hierarchical cluster analysis is based on '1-pearson correlation' distances and ward linkage.

Two-class tests are based on Student's $t$-test assuming equal variance. Multi-class tests are based on F-tests assuming equal variance. $P$-values are corrected for multiple comparisons (Bonferroni).

For classification, we have fitted a regularized multinomial regression model using LASSO (Least Absolute Shrinkage Selection Operator). ${ }^{33}$ The advantage of this classification approach is that a feature selection step is included, in which the effects of most microRNAs are estimated to be exactly zero, thereby significantly reducing the number of included microRNAs in the final model without any external feature selection. In a data set 
containing a lot of covariates, LASSO can help to select a set of covariates that provide an efficient prediction. The complexity of the fitted model is controlled by the penalty factor $\lambda$. The lower it is, the lower the penalty, resulting in more complex models, that is, more microRNAs (features included). Thus, $-\log \lambda$ is used as a measure of model complexity. Classification performance was estimated by 10 -fold cross-validation repeated 10 times. For each 10-fold cross-validation, the data set was split randomly into 10 equally sized test sets, while the remaining samples were used for model fitting. A performance graph showing sensitivity, positive predictive value and accuracy as a function of model complexity (number of microRNAs in the model) can be a help to select the most efficient model.

In the attempt to use microRNA expression profiles to subclassification of ampullary adenocarcinomas in pancreatobiliary and intestinal-like adenocarcinomas, we tried two different models. First, each of the ampullary cancer samples were compared with pancreatic and duodenal adenocarcinoma samples in terms of Pearson correlations (maximum average correlation). Then another LASSO classifier, using $10 \times 5$-fold cross-validation, was fitted to separate ampullary adenocarcinomas into pancreatobiliary type or intestinal type with an expression profile more like duodenal adenocarcinoma. The ampullary adenocarcinoma samples were not used to fit the classifier, thus the classifier was made blind to the results of the histologically and immunohistochemically classification. Each sample was scored using the pancreatic vs duodenal adenocarcinoma classifier. If the pancreatic adenocarcinoma class score was $>0.5$ (the duodenal class score was $<0.5$ ) the sample was classified as pancreatobiliary type.

\section{Results}

RNA extraction was satisfying in all samples (mean $260 / 280 \mathrm{~nm}$ absorbance ratio $=1.85$ ). After exclusion of outliers (10 pancreatic adenocarcinomas; 11 ampullary adenocarcinomas), the final data set was pre-processed as described in 'Materials and methods', resulting in a data set comprising 307 samples (pancreatic adenocarcinomas $n=160$, ampullary adenocarcinomas $n=96$, chronic pancreatitis $n=23$ and normal $n=28$ ) and 475 microRNAs for statistical analysis. Outliers were distributed between tumor blocks of all ages, and no clustering related to the age of tumor blocks was found.

\section{MicroRNA Expression Patterns in Pancreatic Cancer and Controls}

We compared microRNA profiles of pancreatic adenocarcinomas, ampullary adenocarcinomas, chronic pancreatitis and normal pancreas using class comparison analysis. Table 2 shows all microRNAs significantly differentially expressed in the tissue-class comparison analysis.

Pancreatic adenocarcinomas vs normal pancreas. Eighty-four miRNAs were differentially expressed between pancreatic adenocarcinoma and normal pancreas (43 microRNAs at higher level in adenocarcinoma; 41 microRNAs at lower level in adenocarcinoma) $(P<0.05)$. Some selected microRNAs (selection based on $P$-values, $d m$-values, fold change and novelty) and comparison with other studies are shown in Table 3. Nine of the significantly differentially expressed miRNAs described by Bloomston et $a l^{20}$ were also found in our study (miR-21, miR-100*, miR-143/miR-143*, miR-148a, miR-148a*, miR-205, miR-210, miR-222 and miR375). Eleven of the significantly differentially expressed miRNAs described by Szafranska et $a 1^{21,26}$ were found in our study with very similar $d m$-values and fold change (miR-31, miR-130b, miR-143/miR-143*, miR-148a, miR-196b, miR-205, miR-210, miR-216, miR-217, miR-222/miR-222* and miR-375).

Only seven miRNAs described in pancreatic adenocarcinomas by both Bloomston and Szafranka could not be validated in our study. All miRNAs either up- or down-regulated compared with normal pancreas in other studies ${ }^{20,21,26}$ were also significantly differentially expressed in our study before the strict Bonferonni correction for multiple comparisons, except miR-155 that was not present in our microRNA array (Table 2).

Pancreatic adenocarcinomas vs chronic pancreatitis. Thirty-two microRNAs were differentially expressed between pancreatic adenocarcinomas and pancreatitis (15 microRNAs at higher level in adenocarcinoma; 17 microRNAs at lower level in adenocarcinoma) $(P<0.05)$ (Table 1). Some selected microRNAs (selection based on $P$-values, $d m$-values, fold change and novelty) and comparison with other studies are shown in Table 3. MiR-148a was also found to be significantly down-regulated by Bloomston et $a l^{20}$ Szafranska et $a l^{21,26}$ also found that miR-148a, miR-196b, miR-196a and miR-205 were differentially expressed in pancreatic adenocarcinoma and chronic pancreatitis (Table 3).

Pancreatic adenocarcinomas and chronic pancreatitis vs normal pancreas. MiR-198 and miR-650 had higher expression in both adenocarcinoma and chronic pancreatitis compared with normal pancreas tissue. MiR-130b, miR-141*, miR-194* and miR-219-1-3p had reduced expression in both adenocarcinoma and chronic pancreatitis (Table 2).

Ampullary adenocarcinomas. The microRNA expression profile in ampullary adenocarcinomas was very similar to the pancreatic adenocarcinoma 
Table 2 All microRNAs significantly differently expressed in ductal adenocarcinomas (PDAC), ampullary adenocarcinomas (A-AC), chronic pancreatitis (CP) and normal pancreas (NP)

\begin{tabular}{|c|c|c|c|c|c|}
\hline Normal pancreas vs PDAC & P-value & $\mathrm{dm}^{*}$ & Normal pancreas vs $A-A C$ & P-value & $\mathrm{dm} *$ \\
\hline hsa-miR-198 & $1.222 \times 10^{-38}$ & 4.95 & has-miR-198 & $1.071 \times 10^{-27}$ & 4.28 \\
\hline hsa-miR-34c-5p & $1.301 \times 10^{-37}$ & 3.11 & has-miR-10a & $2.530 \times 10^{-27}$ & 2.11 \\
\hline hsa-miR-21 & $2.409 \times 10^{-27}$ & 2.13 & has-miR-650 & $3.280 \times 10^{-27}$ & 4.34 \\
\hline hsa-miR-708 & $1.205 \times 10^{-26}$ & 2.19 & hsa-miR-34c-5p & $5.508 \times 10^{-26}$ & 2.69 \\
\hline hsa-miR-614 & $3.421 \times 10^{-24}$ & 5.60 & hsa-miR-30a* & $1.434 \times 10^{-25}$ & -2.42 \\
\hline hsa-miR-196b & $5.861 \times 10^{-24}$ & 4.40 & hsa-miR-492 & $8.001 \times 10^{-25}$ & 8.58 \\
\hline hsa-miR-939 & $3.690 \times 10^{-23}$ & 3.00 & hsa-miR-148a & $8.210 \times 10^{-24}$ & -3.60 \\
\hline hsa-miR-148a & $3.785 \times 10^{-23}$ & -3.72 & hsa-miR-30e* & $1.480 \times 10^{-23}$ & -2.24 \\
\hline hsa-miR-801 & $4.942 \times 10^{-22}$ & 2.95 & hsa-miR-801 & $2.752 \times 10^{-23}$ & 3.32 \\
\hline hsa-miR-886-5p & $5.080 \times 10^{-22}$ & 2.21 & hsa-miR-614 & $8.103 \times 10^{-23}$ & 6.24 \\
\hline hsa-miR-210 & $1.728 \times 10^{-21}$ & 2.31 & hsa-miR-649 & $4.450 \times 10^{-22}$ & 4.31 \\
\hline hsa-miR-190b & $3.789 \times 10^{-21}$ & -3.00 & hsa-miR-143 & $9.547 \times 10^{-22}$ & 2.06 \\
\hline hsa-miR-142-3p & $9.153 \times 10^{-21}$ & 2.17 & hsa-miR-323-3p & $2.533 \times 10^{-21}$ & -2.02 \\
\hline hsa-miR-130b* & $2.347 \times 10^{-20}$ & -3.99 & hsa-miR-939 & $3.711 \times 10^{-21}$ & 3.07 \\
\hline hsa-miR-649 & $3.987 \times 10^{-20}$ & 3.73 & hsa-miR-130b* & $1.044 \times 10^{-20}$ & -3.52 \\
\hline hsa-miR-30a* & $3.459 \times 10^{-19}$ & -2.00 & hsa-miR-335 & $1.115 \times 10^{-20}$ & -2.26 \\
\hline hsa-miR-650 & $1.272 \times 10^{-18}$ & 3.47 & hsa-miR-30c & $1.195 \times 10^{-20}$ & -2.07 \\
\hline hsa-miR-492 & $6.125 \times 10^{-18}$ & 7.38 & hsa-miR-31 & $1.590 \times 10^{-20}$ & 3.19 \\
\hline hsa-miR-922 & $7.592 \times 10^{-18}$ & 3.99 & hsa-miR-147b & $2.942 \times 10^{-20}$ & 4.44 \\
\hline hsa-miR-31 & $1.992 \times 10^{-17}$ & 2.99 & hsa-miR-130b & $4.314 \times 10^{-20}$ & -2.82 \\
\hline hsa-miR-219-1-3p & $1.036 \times 10^{-16}$ & -4.15 & hsa-miR-210 & $7.733 \times 10^{-20}$ & 2.16 \\
\hline hsa-miR-432* & $1.201 \times 10^{-16}$ & -4.29 & hsa-miR-922 & $1.090 \times 10^{-19}$ & 4.38 \\
\hline hsa-miR-130b & $1.739 \times 10^{-16}$ & -2.63 & hsa-miR-622 & $2.175 \times 10^{-19}$ & 3.23 \\
\hline hsa-miR-100* & $3.505 \times 10^{-16}$ & 2.75 & hsa-miR-548b-5p & $2.684 \times 10^{-19}$ & 2.17 \\
\hline hsa-miR-222* & $3.959 \times 10^{-16}$ & 2.10 & hsa-miR-142-3p & $2.896 \times 10^{-19}$ & 2.21 \\
\hline hsa-miR-375 & $2.059 \times 10^{-15}$ & -3.23 & hsa-miR-891a & $2.009 \times 10^{-18}$ & -6.18 \\
\hline hsa-miR-135b* & $2.364 \times 10^{-15}$ & 3.45 & hsa-miR-196b & $6.028 \times 10^{-18}$ & 5.09 \\
\hline hsa-miR-592 & $2.783 \times 10^{-15}$ & -2.14 & hsa-miR-135b* & $9.602 \times 10^{-18}$ & 4.06 \\
\hline hsa-miR-494 & $3.180 \times 10^{-15}$ & 2.08 & hsa-miR-133b & $1.266 \times 10^{-17}$ & 2.64 \\
\hline hsa-miR-148a * & $3.377 \times 10^{-15}$ & -5.17 & hsa-miR-590-5p & $1.715 \times 10^{-17}$ & -2.14 \\
\hline hsa-miR-635 & $5.813 \times 10^{-15}$ & 2.57 & hsa-miR-494 & $4.378 \times 10^{-17}$ & 2.37 \\
\hline hsa-miR-598 & $5.931 \times 10^{-15}$ & -2.14 & hsa-miR-432* & $1.070 \times 10^{-16}$ & -4.52 \\
\hline hsa-miR-622 & $2.206 \times 10^{-14}$ & 2.49 & hsa-miR-133a & $1.193 \times 10^{-16}$ & 2.46 \\
\hline hsa-miR-877 & $4.509 \times 10^{-13}$ & 2.01 & hsa-miR-190b & $4.543 \times 10^{-16}$ & -2.88 \\
\hline hsa-miR-875-5p & $1.180 \times 10^{-12}$ & 2.04 & hsa-miR-135b & $1.131 \times 10^{-15}$ & 2.14 \\
\hline hsa-miR-451 & $1.863 \times 10^{-12}$ & 2.53 & hsa-miR-548d-5p & $1.330 \times 10^{-15}$ & 2.02 \\
\hline hsa-miR-891a & $2.297 \times 10^{-12}$ & -4.88 & hsa-miR-598 & $1.460 \times 10^{-15}$ & -2.48 \\
\hline hsa-miR-509-5p & $3.820 \times 10^{-12}$ & 3.91 & hsa-miR-923 & $3.897 \times 10^{-15}$ & 2.25 \\
\hline hsa-miR-518d-3p & $1.096 \times 10^{-11}$ & -3.94 & hsa-miR-143* & $9.515 \times 10^{-15}$ & 6.89 \\
\hline hsa-miR-648 & $2.151 \times 10^{-11}$ & 3.11 & hsa-miR-604 & $1.098 \times 10^{-14}$ & 2.18 \\
\hline hsa-miR-449b & $2.229 \times 10^{-11}$ & -2.73 & hsa-miR-148a* & $2.172 \times 10^{-14}$ & -4.36 \\
\hline hsa-miR-141* & $3.964 \times 10^{-11}$ & -2.64 & hsa-miR-411* & $3.799 \times 10^{-14}$ & -4.10 \\
\hline hsa-miR-643 & $4.665 \times 10^{-11}$ & 2.59 & hsa-miR-7-2* & $2.883 \times 10^{-13}$ & -5.07 \\
\hline hsa-miR-575 & $6.107 \times 10^{-11}$ & 2.91 & hsa-miR-551b* & $5.218 \times 10^{-13}$ & 2.05 \\
\hline hsa-miR-193b* & $6.689 \times 10^{-11}$ & -3.04 & hsa-miR-644 & $6.766 \times 10^{-13}$ & 3.60 \\
\hline hsa-miR-217 & $1.017 \times 10^{-10}$ & -5.37 & hsa-miR-379* & $1.225 \times 10^{-12}$ & -3.95 \\
\hline hsa-miR-154* & $1.553 \times 10^{-10}$ & -3.49 & hsa-miR-639 & $3.556 \times 10^{-12}$ & 2.88 \\
\hline hsa-miR-34b* & $1.927 \times 10^{-10}$ & 2.66 & hsa-miR-643 & $4.604 \times 10^{-12}$ & 2.46 \\
\hline hsa-miR-7-2* & $3.445 \times 10^{-10}$ & -3.88 & hsa-miR-487b & $5.427 \times 10^{-12}$ & -2.21 \\
\hline hsa-miR-147b & $9.704 \times 10^{-10}$ & 3.37 & hsa-miR-575 & $5.462 \times 10^{-12}$ & 3.28 \\
\hline hsa-miR-584 & $1.092 \times 10^{-09}$ & 3.38 & hsa-miR-375 & $6.319 \times 10^{-12}$ & -2.76 \\
\hline hsa-miR-449a & $1.253 \times 10^{-09}$ & -2.61 & hsa-miR-635 & $7.068 \times 10^{-12}$ & 2.84 \\
\hline hsa-miR-411* & $1.629 \times 10^{-09}$ & -3.02 & hsa-miR-187 & $8.352 \times 10^{-12}$ & 6.23 \\
\hline hsa-miR-589* & $1.692 \times 10^{-09}$ & -3.56 & hsa-miR-875-5p & $2.504 \times 10^{-11}$ & 2.25 \\
\hline hsa-miR-216b & $5.230 \times 10^{-09}$ & -5.47 & hsa-miR-154* & $1.064 \times 10^{-10}$ & -3.74 \\
\hline hsa-miR-379* & $5.579 \times 10^{-09}$ & -2.94 & hsa-miR-888 & $1.600 \times 10^{-10}$ & -3.34 \\
\hline hsa-miR-216a & $6.104 \times 10^{-09}$ & -4.67 & hsa-miR-937 & $2.445 \times 10^{-10}$ & 3.68 \\
\hline hsa-miR-219-5p & $7.613 \times 10^{-09}$ & -2.92 & hsa-miR-203 & $3.011 \times 10^{-10}$ & 2.01 \\
\hline hsa-miR-486-3p & $1.286 \times 10^{-08}$ & -3.05 & hsa-miR-449b & $5.703 \times 10^{-10}$ & -2.79 \\
\hline hsa-miR-153 & $1.457 \times 10^{-08}$ & -2.83 & hsa-miR-640 & $6.305 \times 10^{-10}$ & 3.22 \\
\hline hsa-miR-143* & $3.768 \times 10^{-08}$ & 4.82 & hsa-miR-147 & $9.659 \times 10^{-10}$ & 3.65 \\
\hline hsa-miR-542-5p & $5.920 \times 10^{-08}$ & 2.45 & hsa-miR-518d-3p & $1.098 \times 10^{-09}$ & -3.71 \\
\hline hsa-miR-644 & $6.572 \times 10^{-08}$ & 2.72 & hsa-miR-648 & $1.135 \times 10^{-09}$ & 2.95 \\
\hline hsa-miR-944 & $7.423 \times 10^{-08}$ & 3.15 & hsa-miR-33a* & $1.502 \times 10^{-09}$ & -2.411 \\
\hline hsa-miR-129-5p & $7.576 \times 10^{-08}$ & -3.29 & hsa-miR-656 & $1.778 \times 10^{-09}$ & -2.31 \\
\hline hsa-miR-19a* & $1.087 \times 10^{-07}$ & -2.69 & hsa-miR-129-3p & $2.077 \times 10^{-09}$ & -4.66 \\
\hline hsa-miR-377* & $2.792 \times 10^{-07}$ & -2.72 & hsa-miR-217 & $2.684 \times 10^{-09}$ & -5.61 \\
\hline hsa-miR-640 & $3.933 \times 10^{-07}$ & 2.85 & hsa-miR-153 & $2.810 \times 10^{-09}$ & -3.33 \\
\hline
\end{tabular}


Table 2 (Continued)

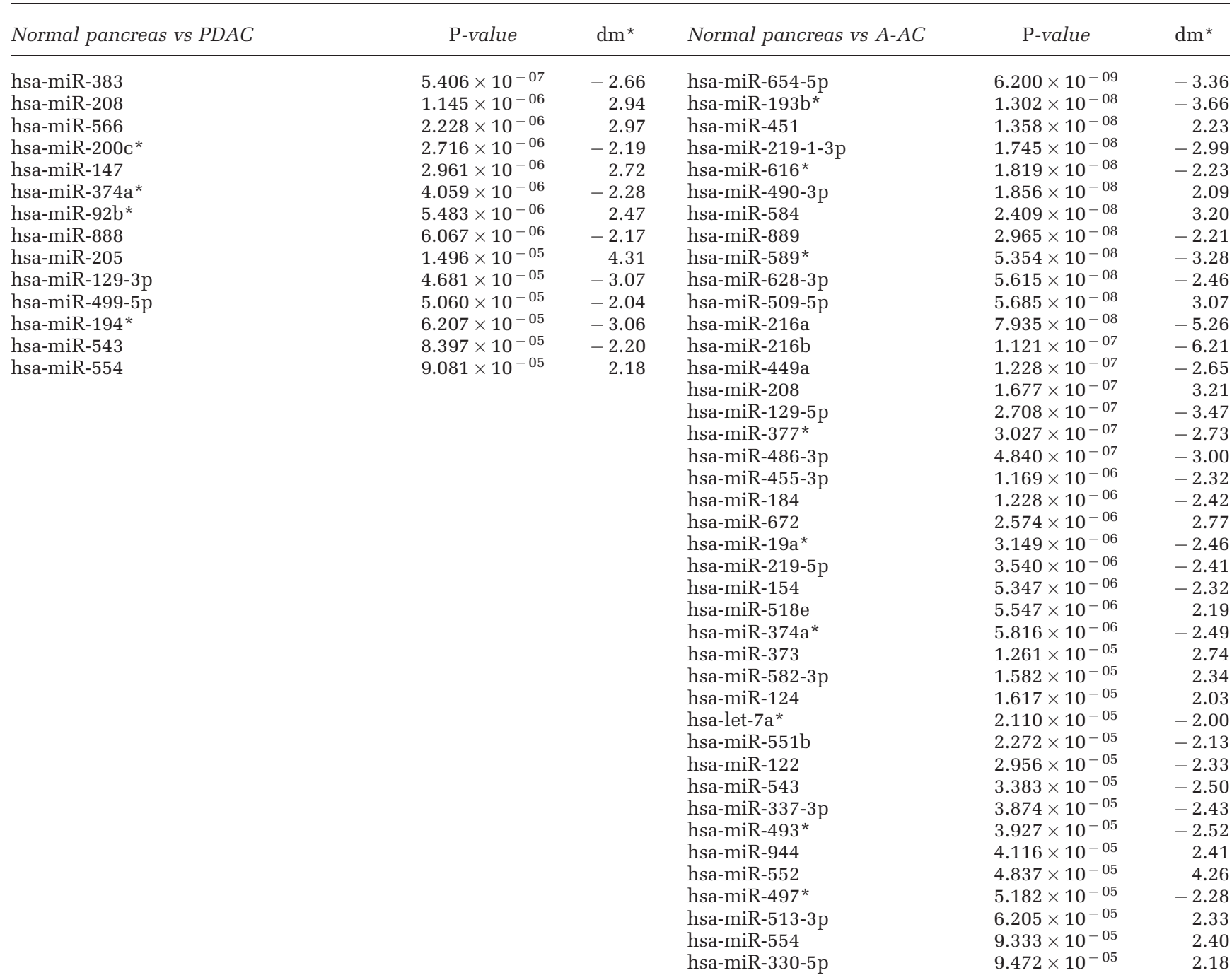

\begin{tabular}{|c|c|c|c|c|c|}
\hline Chronic pancreatitis vs $P D A C$ & $\mathrm{P}$-value & $\mathrm{dm}^{*}$ & Chronic pancreatitis vs $A-A C$ & $\mathrm{P}$-value & $\mathrm{dm}^{*}$ \\
\hline hsa-miR-614 & $5.690 \times 10^{-19}$ & 5.06 & has-miR-492 & $1.396 \times 10^{-21}$ & 8.58 \\
\hline hsa-miR-492 & $2.343 \times 10^{-15}$ & 7.38 & has-miR-622 & $2.612 \times 10^{-20}$ & 3.52 \\
\hline hsa-miR-622 & $2.622 \times 10^{-15}$ & 2.78 & has-miR-614 & $6.228 \times 10^{-19}$ & 5.70 \\
\hline hsa-miR-135b* & $1.635 \times 10^{-13}$ & 3.46 & hsa-miR-147b & $6.070 \times 10^{-16}$ & 4.38 \\
\hline hsa-miR-196b & $9.787 \times 10^{-13}$ & 3.06 & hsa-miR-135b* & $6.091 \times 10^{-16}$ & 4.07 \\
\hline hsa-miR-198 & $2.292 \times 10^{-12}$ & 2.39 & hsa-miR-215 & $6.331 \times 10^{-15}$ & 3.04 \\
\hline hsa-miR-516a-3p & $2.349 \times 10^{-09}$ & 2.06 & hsa-miR-194* & $1.470 \times 10^{-13}$ & 6.68 \\
\hline hsa-miR-122 & $5.639 \times 10^{-09}$ & -3.79 & hsa-miR-135b & $8.533 \times 10^{-13}$ & 2.03 \\
\hline hsa-miR-509-5p & $1.198 \times 10^{-08}$ & 3.33 & hsa-miR-203 & $4.425 \times 10^{-12}$ & 2.66 \\
\hline hsa-miR-147b & $7.185 \times 10^{-08}$ & 3.31 & hsa-miR-194 & $5.837 \times 10^{-12}$ & 2.34 \\
\hline hsa-miR-148a & $1.331 \times 10^{-07}$ & -2.10 & hsa-miR-192 & $9.373 \times 10^{-12}$ & 2.24 \\
\hline hsa-miR-648 & $1.848 \times 10^{-07}$ & 2.57 & hsa-miR-516a-3p & $1.293 \times 10^{-11}$ & 2.05 \\
\hline hsa-miR-643 & $3.758 \times 10^{-07}$ & 2.03 & hsa-miR-133a & $3.839 \times 10^{-11}$ & 2.06 \\
\hline hsa-miR-125b-2 * & $6.382 \times 10^{-07}$ & -2.24 & hsa-miR-196b & $1.213 \times 10^{-10}$ & 3.76 \\
\hline hsa-miR-432* & $7.176 \times 10^{-07}$ & -2.70 & hsa-miR-891a & $2.033 \times 10^{-10}$ & -4.82 \\
\hline hsa-miR-575 & $1.937 \times 10^{-06}$ & 2.27 & hsa-miR-133b & $4.165 \times 10^{-10}$ & -2.07 \\
\hline hsa-miR-520c-3p & $2.581 \times 10^{-06}$ & 2.21 & hsa-miR-649 & $8.478 \times 10^{-10}$ & 2.41 \\
\hline hsa-miR-584 & $2.746 \times 10^{-06}$ & 2.77 & hsa-miR-654-5p & $9.579 \times 10^{-10}$ & -3.95 \\
\hline hsa-miR-377* & $2.853 \times 10^{-06}$ & -2.69 & hsa-miR-122 & $9.937 \times 10^{-10}$ & -3.96 \\
\hline hsa-miR-148a* & $3.135 \times 10^{-06}$ & -3.24 & hsa-miR-411* & $1.463 \times 10^{-09}$ & -3.44 \\
\hline hsa-miR-891a & $3.482 \times 10^{-06}$ & -3.52 & hsa-miR-125b-2* & $2.103 \times 10^{-09}$ & -3.23 \\
\hline hsa-miR-337-3p & $4.109 \times 10^{-06}$ & -2.99 & hsa-miR-490-3p & $3.391 \times 10^{-09}$ & 2.67 \\
\hline hsa-miR-154* & $7.751 \times 10^{-06}$ & -2.69 & hsa-miR-379* & $4.442 \times 10^{-09}$ & -3.38 \\
\hline hsa-miR-379* & $9.203 \times 10^{-06}$ & -2.38 & hsa-miR-187 & $5.383 \times 10^{-08}$ & 5.39 \\
\hline
\end{tabular}


Table 2 (Continued)

\begin{tabular}{|c|c|c|c|c|c|}
\hline Chronic pancreatitis vs PDAC & P-value & $\mathrm{dm}^{*}$ & Chronic pancreatitis vs $A-A C$ & $\mathrm{P}$-value & $\mathrm{dm}^{*}$ \\
\hline hsa-miR-411* & $1.129 \times 10^{-05}$ & -2.36 & hsa-miR-450b-5p & $5.835 \times 10^{-08}$ & -3.24 \\
\hline hsa-miR-205 & $1.739 \times 10^{-05}$ & 4.68 & hsa-miR-7-2* & $7.857 \times 10^{-08}$ & -3.88 \\
\hline hsa-miR-208 & $1.995 \times 10^{-05}$ & 2.85 & hsa-miR-656 & $1.063 \times 10^{-07}$ & -2.25 \\
\hline hsa-miR-493* & $4.226 \times 10^{-05}$ & -2.57 & hsa-miR-337-3p & $1.396 \times 10^{-07}$ & -3.52 \\
\hline hsa-miR-7-2* & $4.232 \times 10^{-05}$ & -2.69 & hsa-miR-575 & $1.506 \times 10^{-07}$ & 2.64 \\
\hline hsa-miR-512-3p & $4.673 \times 10^{-05}$ & 2.14 & hsa-miR-432* & $1.806 \times 10^{-07}$ & -2.92 \\
\hline hsa-miR-193b* & $5.576 \times 10^{-05}$ & -2.04 & hsa-miR-493* & $2.734 \times 10^{-07}$ & -3.47 \\
\hline \multirow[t]{4}{*}{ hsa-miR-374a* } & $9.703 \times 10^{-05}$ & -2.11 & hsa-miR-937 & $3.739 \times 10^{-07}$ & 3.21 \\
\hline & & & hsa-miR-888 & $7.074 \times 10^{-07}$ & -2.74 \\
\hline & & & hsa-miR-376b & $1.137 \times 10^{-06}$ & -3.03 \\
\hline & & & hsa-miR-520c-3p & $1.138 \times 10^{-06}$ & 2.35 \\
\hline$P D A C$ vs $A-A C$ & P-value & $\mathrm{dm}$ & hsa-miR-497* & $1.304 \times 10^{-06}$ & -2.90 \\
\hline hsa-miR-194* & $1.849 \times 10^{-24}$ & 5.47 & hsa-miR-518e & $1.643 \times 10^{-06}$ & 2.60 \\
\hline hsa-miR-187 & $1.542 \times 10^{-13}$ & 4.73 & hsa-miR-129-3p & $1.679 \times 10^{-06}$ & -4.02 \\
\hline hsa-miR-654-5p & $2.233 \times 10^{-09}$ & -2.09 & hsa-miR-512-3p & $1.764 \times 10^{-06}$ & 2.60 \\
\hline hsa-miR-552 & $5.041 \times 10^{-09}$ & 3.11 & hsa-miR-648 & $1.974 \times 10^{-06}$ & 2.41 \\
\hline \multirow[t]{5}{*}{ hsa-miR-205 } & $4.694 \times 10^{-05}$ & -2.62 & hsa-miR-639 & $2.346 \times 10^{-06}$ & 2.14 \\
\hline & & & hsa-miR-377* & $2.522 \times 10^{-06}$ & -2.67 \\
\hline & & & hsa-miR-154* & $4.373 \times 10^{-06}$ & -2.94 \\
\hline & & & hsa-miR-208 & $4.534 \times 10^{-06}$ & 3.12 \\
\hline & & & hsa-miR-143* & $4.735 \times 10^{-06}$ & 4.35 \\
\hline \multirow[t]{2}{*}{ Normal pancreas vs chronic pancreatitis } & P-value & $\mathrm{dm}$ & hsa-miR-635 & $5.627 \times 10^{-06}$ & 2.03 \\
\hline & & & hsa-miR-644 & $6.208 \times 10^{-06}$ & 2.34 \\
\hline hsa-miR-194* & $2.106 \times 10^{-07}$ & -4.27 & hsa-miR-147 & $9.783 \times 10^{-06}$ & 2.92 \\
\hline hsa-miR-141* & $1.298 \times 10^{-06}$ & -2.26 & hsa-miR-509-5p & $1.257 \times 10^{-05}$ & 2.48 \\
\hline hsa-miR-198 & $3.891 \times 10^{-06}$ & 2.57 & hsa-miR-518f & $1.312 \times 10^{-05}$ & 2.28 \\
\hline hsa-miR-130b* & $6.243 \times 10^{-06}$ & -2.08 & hsa-miR-922 & $1.542 \times 10^{-05}$ & 2.23 \\
\hline hsa-miR-650 & $1.074 \times 10^{-05}$ & 2.74 & hsa-miR-584 & $1.695 \times 10^{-05}$ & $2.5 \mathrm{c}$ \\
\hline hsa-miR-219-1-3p & $4.458 \times 10^{-05}$ & -2.62 & hsa-miR-148a* & $4.588 \times 10^{-05}$ & -2.44 \\
\hline \multirow[t]{3}{*}{ hsa-miR-766 } & $4.639 \times 10^{-05}$ & 2.56 & hsa-miR-552 & $6.004 \times 10^{-05}$ & 4.62 \\
\hline & & & hsa-miR-154 & $7.767 \times 10^{-05}$ & -2.10 \\
\hline & & & hsa-miR-543 & $1.014 \times 10^{-04}$ & -2.46 \\
\hline
\end{tabular}

$P$-values in this table are not corrected for multiple testing, but only microRNAs significantly expressed $(P<0.05)$ after Bonferroni correction are included.

${ }^{*}$ The difference of means $(\mathrm{dm})$ is the difference between class means (1st tissue-2nd tissue mean $C_{\mathrm{t}}$-values). If the value is positive it means that the average $C_{\mathrm{t}}$ is higher in the 1st tissue class and thus the microRNA is expressed at lower levels in the 1st class. If $d m=1$, then the microRNA is two times up-regulated in the 2nd tissue compared with the 1st tissue. If $d m=-1$, then the microRNA is two times down-regulated in the 2 nd tissue compared with the 1st tissue.

expression profile with an average Pearson correlation of 0.99 between the two tissue classes for the microRNAs passing QC. Only five microRNAs were significantly differentially expressed between these two tissue types (miR-187, miR-194*, miR-205, miR-552, miR-654-5p). The results of the tissue-class comparison analysis for ampullary adenocarcinomas and pancreatic adenocarcinomas compared with chronic pancreatitis and normal pancreas were almost identical. One hundred and ten microRNAs were differentially expressed between ampullary adenocarcinomas and normal pancreas (55 microRNAs at higher level in adenocarcinoma; 55 microRNAs at lower level, Table 2).

KRAS mutational status. Six microRNAs were significantly differentially expressed $(P<0.0001)$ between KRAS mutated and KRAS wild-type tumor samples (miR-10a, miR-135b, miR-135b*, miR-148a, miR-190b, miR-222). Best illustrated for miR-148a and miR-190b where the $C_{\mathrm{t}}$-density curves have distinct peaks depending on KRAS status.

Tumor stage. We could not demonstrate a reliable microRNA profile to separate cancer samples according to tumor stage and lymph node metastasis.

\section{A microRNA Classifier to Distinguish Pancreatic Cancer Samples from Controls}

Figure 1 shows strip charts of $C_{\mathrm{t}}$-values for the 19 most significantly expressed microRNAs when comparing pancreatic adenocarcinomas, ampullary adenocarcinomas, chronic pancreatitis and normal pancreas samples (F-test). The overlap of the $C_{\mathrm{t}^{-}}$ values between different types of tissues $\left(C_{\mathrm{t}}\right.$-density plots, Supplementary Figure S1) illustrates the necessity of combining different microRNAs in a panel to separate cancer samples from pancreatitis and normal pancreas. For this purpose, we used a LASSO classifier with $10 \times 10$-fold cross-validation. 
Table 3 Selected microRNAs significantly differently expressed in pancreatic ductal adenocarcinoma (PDAC), chronic pancreatitis (CP) and normal pancreas (NP)

\begin{tabular}{|c|c|c|c|c|c|c|}
\hline & $\begin{array}{l}\text { Corrected } \\
\text { P-value }\end{array}$ & $\operatorname{Dm}\left(\Delta \Delta \mathrm{C}_{t}\right)^{\mathrm{a}}$ & $\begin{array}{c}\text { Fold } \\
\text { change }^{\mathrm{a}}\end{array}$ & $\begin{array}{l}\text { Bloomston et } \mathrm{al}^{20} \\
\quad \text { fold change }\end{array}$ & $\begin{array}{l}\text { Szafranska } \\
\text { et } \mathrm{al}^{26} \Delta \Delta \mathrm{C}_{t}\end{array}$ & $\begin{array}{l}\text { Szafranska } \\
\text { et } \mathrm{al}^{21} \Delta \mathrm{h}^{\mathrm{b}}\end{array}$ \\
\hline \multicolumn{7}{|c|}{ Normal pancreas vs $P D A C$} \\
\hline hsa-miR-198 & $5.803 \times 10^{-36}$ & 4.95 & 30.98 & & & \\
\hline hsa-miR-34c-5p & $6.182 \times 10^{-35}$ & 3.11 & 8.66 & & & \\
\hline hsa-miR-21 & $1.144 \times 10^{-24}$ & 2.13 & 4.39 & 3.08 & & \\
\hline hsa-miR-614 & $1.625 \times 10^{-21}$ & 5.60 & 48.35 & & & \\
\hline hsa-miR-196b & $2.784 \times 10^{-21}$ & 4.40 & 21.06 & & miR-196a, 6.59 & 2.67 \\
\hline hsa-miR-148a & $1.798 \times 10^{-20}$ & -3.72 & 0.08 & 0.18 & -6.15 & -3.30 \\
\hline hsa-miR-210 & $8.209 \times 10^{-19}$ & 2.31 & 4.96 & 2.97 & 2.31 & 2.82 \\
\hline hsa-miR-492 & $6.120 \times 10^{-18}$ & 7.38 & 166.99 & & & \\
\hline hsa-miR-31 & $9.463 \times 10^{-15}$ & 2.99 & 7.94 & & 2.48 & 2.79 \\
\hline hsa-miR-130b & $8.260 \times 10^{-14}$ & -2.63 & 0.16 & & -3.86 & -2.43 \\
\hline hsa-miR-100* & $1.665 \times 10^{-13}$ & 2.75 & 6.70 & 2.49 & & \\
\hline hsa-miR-222* & $1.881 \times 10^{-13}$ & 2.10 & 4.29 & 2.70 & & $\operatorname{miR}-222,2.06$ \\
\hline hsa-miR-375 & $9.778 \times 10^{-13}$ & -3.23 & 0.11 & 0.46 & -6.20 & -2.51 \\
\hline hsa-miR-148a* & $1.604 \times 10^{-12}$ & -5.17 & 0.03 & 0.18 & & \\
\hline hsa-miR-141* & $1.883 \times 10^{-8}$ & -2.64 & 0.16 & & & $\operatorname{miR}-141,-1.50$ \\
\hline hsa-miR-217 & $4.833 \times 10^{-8}$ & -5.37 & 0.02 & & -10.81 & -5.68 \\
\hline hsa-miR-216b & $2.484 \times 10^{-6}$ & -5.47 & 0.02 & & & miR-216, -5.45 \\
\hline hsa-miR-216a & $2.899 \times 10^{-6}$ & -4.67 & 0.04 & & & $\operatorname{miR}-216,-5.45$ \\
\hline hsa-miR-143* & $1.790 \times 10^{-5}$ & 4.82 & 28.25 & $\operatorname{miR}-143,2.19$ & $\operatorname{miR}-143,1.36$ & miR-143, 1.94 \\
\hline hsa-miR-205 & $7.105 \times 10^{-3}$ & 4.31 & 19.90 & 2.24 & 1.70 & 2.22 \\
\hline hsa-miR-194* & 0.029 & -3.06 & 0.12 & & & \\
\hline hsa-miR-96 & c & & & & -4.35 & -1.64 \\
\hline hsa-miR-145 & c & & & & 1.56 & 1.83 \\
\hline hsa-miR-146a & c & & & $\operatorname{miR}-146,2.44$ & & 2.09 \\
\hline hsa-miR-148b & c & & & 0.31 & & -2.29 \\
\hline hsa-miR-221 & c & & & 3.41 & & 1.48 \\
\hline hsa-miR-223 & c & & & 2.53 & 2.22 & 2.39 \\
\hline hsa-miR-155 & & Not measured & & 2.10 & 3.02 & 2.34 \\
\hline \multicolumn{7}{|c|}{ Chronic pancreatitis vs PDAC } \\
\hline hsa-miR-614 & $2.703 \times 10^{-16}$ & 5.06 & 33.31 & & & \\
\hline hsa-miR-492 & $1.113 \times 10^{-12}$ & 7.38 & 166.23 & & & \\
\hline hsa-miR-622 & $1.245 \times 10^{-12}$ & 2.78 & 6.86 & & & \\
\hline hsa-miR-135b* & $7.767 \times 10^{-11}$ & 3.46 & 11.02 & & & \\
\hline hsa-miR-196b & $4.649 \times 10^{-10}$ & 3.06 & 8.36 & & miR-196a, 4.69 & 1.64 \\
\hline has-miR-198 & $1.089 \times 10^{-9}$ & 2.39 & 5.22 & & & \\
\hline hsa-miR-148a & $6.323 \times 10^{-5}$ & -2.10 & 0.23 & 0.22 & -4.28 & -1.98 \\
\hline hsa-miR-205 & $8.262 \times 10^{-3}$ & 4.68 & 25.59 & & 3.34 & \\
\hline hsa-miR-96 & NS & & & 1.77 & $-1.70^{\mathrm{d}}$ & \\
\hline hsa-miR-375 & NS & & & 0.46 & -4.05 & -1.73 \\
\hline hsa-miR-155 & Not measured & 1.88 & 1.80 & 1.38 & & \\
\hline
\end{tabular}

$P$-values are Bonferroni corrected for multiple testing. $P$-values, $d m$ and fold change for the microRNAs that are also found significantly differently expressed by Bloomston et $a l^{20}$ and Szafranska et $a l^{21,26}$ are listed.

aThe difference of means $(\mathrm{dm})$ column corresponds to the log2 fold change and is the difference between class means (1st tissue - 2nd tissue mean $C_{\mathrm{t}}$-values). If the value is positive, it means that the average $C_{\mathrm{t}}$ is higher in the 1st tissue class and thus the microRNA is expressed at lower levels in the 1st class. If $d m=1$, then the microRNA is two times up-regulated in the 2nd tissue compared with the 1 st tissue. If $d m=-1$, then the microRNA is two times down-regulated in the 2nd tissue compared with the 1st tissue. The fold change is $2^{\Delta \Delta C_{\mathrm{t}}}$ for qPCR data.

${ }^{\mathrm{b}} \Delta h$ corresponds to the $d m$-value used in our study and the other study by Szafranska et al.

${ }^{\mathrm{C}}$ Loss of significance after Bonferroni correction.

${ }^{\mathrm{d}}$ Borderline significant. NS, not significant.

The classifier performance graph showing sensitivity, positive predictive value and accuracy for a given model complexity is illustrated in Figure 2. An average prediction accuracy of 0.98 was obtained at a complexity of 4.2. When examining the classification performance graph it appeared slightly over-fitted at the high model complexities. Therefore, we estimate that a more robust classifier exists at a model complexity around 3.5 where the curve breaks close to $98 \%$ prediction accuracy. When building a classifier on the full data set with this model complexity, the classifier uses the 19 microRNAs listed in Table 4. This panel of microRNAs separates samples containing pancreatic or ampullary cancer cells from non-neoplastic tissue samples with a sensitivity of 0.985 , a positive predictive value of 0.978 and an accuracy of 0.969 . Supplementary Figure S2 shows a heat-map based on the 19 classifier microRNAs. Fourteen of the 19 microRNAs in this classifier are not included in earlier reports of microRNA profiles in pancreatic cancer tissue (miR-34c-5p, miR-122, miR-135b, 
Tissue comparison sorted by F-test $\mathrm{p}$-value

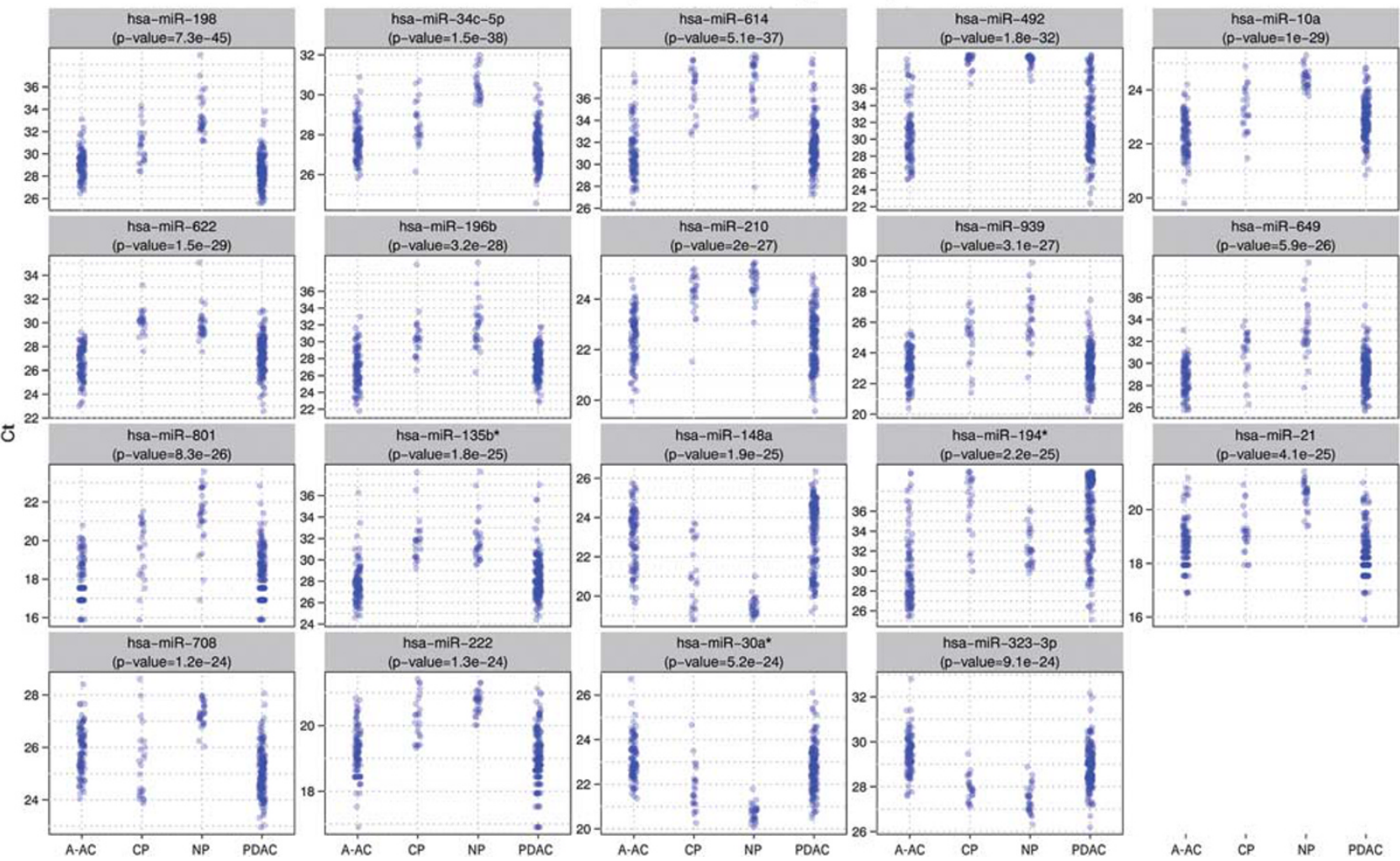

Figure 1 Strip charts showing tissue comparison of microRNAs sorted by F-test, $P$-values. A-AC, ampullary adenocarcinoma; $C P$, chronic pancreatitis; NP, normal pancreas; PDAC, pancreatic ductal adenocarcinomas adenocarcinoma.

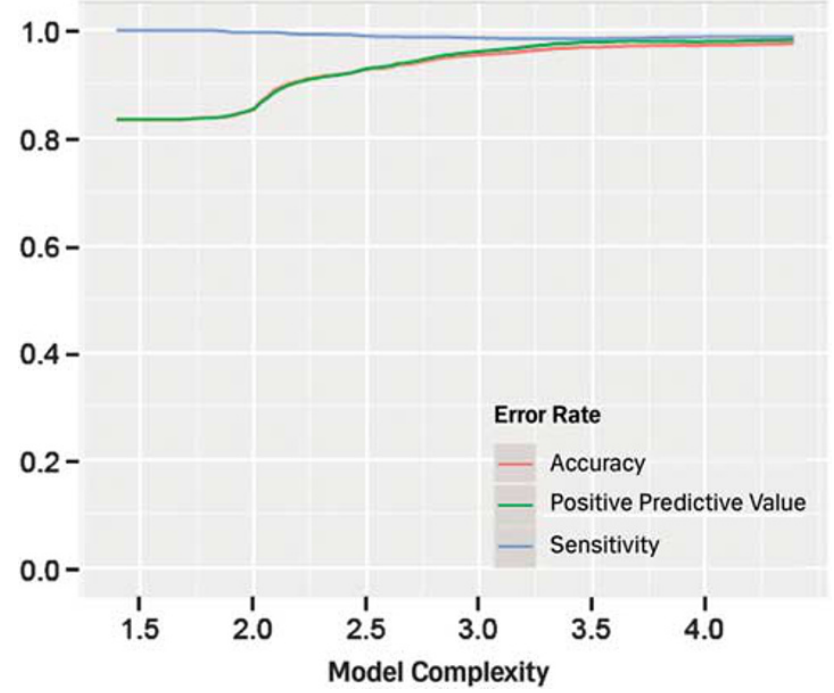

Figure 2 The LASSO classifier performance graph showing sensitivity, positive predictive value and accuracy for a given model complexity. The performance can be increased by increasing the model complexity, that is, more microRNAs (features) included. The Performance graph shows a sensitivity of 0.985 , a positive predictive value of 0.978 and an accuracy of 0.969 with the selected model complexity of 3.5.

miR-135b*, miR-136*, miR-198, miR-451, miR-4903p, miR-492, miR-509-5p, miR-571, miR-614, miR622, miR-939).

\section{Simple Combinations of Two microRNAs to Separate Cancer from Controls}

The difference in each patient's expression of miR$196 \mathrm{~b}$ and miR-217 (ie, the ASURAGEN-test) ${ }^{26}$ in our setting is illustrated in Figure 3a. The Applied Biosystems assay measures only miR-196b, one nucleotide different from miR-196a. These two microRNAs could separate pancreatic ductal adenocarcinomas from chronic pancreatitis $(P=3.52 \times$ $\left.10^{-7}\right)$ and normal pancreas $\left(P=8.59 \times 10^{-20}\right)$. Figure 3b-d illustrate three other combinations of microRNAs (miR-411-miR-198; miR-614-miR-122; miR-614-miR-93*) that performed better than the ASURAGEN-test. The overall best combination to separate pancreatic and ampullary adenocarcinomas from chronic pancreatitis and normal pancreas was the difference between miR-411 and miR-198 $\left(P=4.64 \times 10^{-49}\right)$. The difference in miR-614 and miR-122 was the best combination to separate pancreatic adenocarcinomas from chronic pancreatitis $\left(P=7.76 \times 10^{-18}\right)$ (Table 5).

\section{Subclassification of Ampullary Adenocarcinomas by MicroRNA Expression Profiles}

The 96 ampullary adenocarcinoma samples were subclassified into pancreatobiliary $(n=48)$ or intestinal-type adenocarcinomas $(n=48)$ histologically 
Table 4 LASSO classifier features for a selected model of 19 microRNAs (model complexity of 3.5) and their stability in 1st 10fold cross-validation data set

\begin{tabular}{lc} 
Feature & Stability (1-10) \\
\hline hsa-miR-122 & 6 \\
hsa-miR-135b & 10 \\
hsa-miR-135b* & 10 \\
hsa-miR-136* & 9 \\
hsa-miR-186 & 10 \\
hsa-miR-196b & 10 \\
hsa-miR-198 & 10 \\
hsa-miR-203 & 5 \\
hsa-miR-222 & 10 \\
hsa-miR-23a & 5 \\
hsa-miR-34c-5p & 8 \\
hsa-miR-451 & 10 \\
hsa-miR-490-3p & 5 \\
hsa-miR-492 & 10 \\
hsa-miR-509-5p & 9 \\
hsa-miR-571 & 10 \\
hsa-miR-614 & 10 \\
hsa-miR-622 & 10 \\
hsa-miR-939 & 8
\end{tabular}

The stability measure indicates how certain we are that a microRNA is biologically important and would be a useful biomarker in an independent cohort.

and immunohistochemically by two experienced pathologists (AR,TH). We applied two models for classification by microRNA expression profiles, both were done blinded to the results of the pathologists histological and immunohistochemical classification. For both classifiers we used our 160 pancreatic adenocarcinoma samples and 15 new duodenal adenocarcinoma samples (these were analyzed the same way as the other samples in this study). The ampullary adenocarcinoma samples were not used to fit the classifiers so we did not introduce a bias from the histological subclassification in our classifiers. The microRNA expression profile in all ampullary adenocarcinoma samples was highly correlated to both pancreatic (Pearson correlation $=0.990$ ) and duodenal adenocarcinomas (Pearson correlation $=0.989$ ). When Pearson correlation was used for subclassification of each ampullary adenocarcinoma sample, 53 samples were scored as pancreatic adenocarcinoma and 43 were scored as duodenal adenocarcinoma. Sixty-nine percent of the samples were scored in the same class as by the histological and immunohistochemical classification.

With the LASSO classifier fitted to separate ampullary adenocarcinomas into pancreatobiliary type or intestinal type with an expression profile more like duodenal adenocarcinoma, 79 samples scored as pancreatic adenocarcinoma and 17 scored as duodenal adenocarcinoma. Sixty-eight percent of the samples were scored in the same class as by histological and immunohistochemical classification. Supplementary Figure S3 shows the predicted subclasses of ampullary cancers when they are scored with the LASSO classifier. Supplementary Table S4 gives the 10 best microRNAs to separate pancreatobiliary type and intestinal adenocarcinoma type by the same LASSO classifier. Sixteen samples were classified different than histological and immunohistochemical classification by both methods. Sixty-two percent of the samples were classified in the same subclass by both microRNA expression methods. Supplementary Table S5 shows the subclassification of all ampullary adenocarcinoma samples with different methods.

\section{Discussion}

This is one of the few larger microRNA studies of patients operated for pancreatic and ampullary adenocarcinomas. We used non-microdissected formalin-fixed paraffin-embedded tumor samples and tested 664 miRNAs, including several recently discovered microRNAs. The primary goals were to identify new diagnostic profiles of microRNAs for pancreatic cancer. We present three new combinations of two microRNAs (differences between miR411 and miR-198, miR-614 and miR-122, miR-614 and miR-93*), which strongly discriminate pancreatic adenocarcinoma and ampullary adenocarcinoma tissue from chronic pancreatitis and normal pancreas, without the need of data normalization. Furthermore, the usefulness of the ASURAGEN-test (difference between miR-196b and miR-217) was confirmed in our study. We also present a new diagnostic panel of 19 microRNAs that separates the samples containing cancer cells from non-neoplastic tissue samples with high sensitivity $(98.5 \%)$, positive predictive value (97.8\%) and accuracy (96.9\%). Fourteen of the 19 microRNAs in this classifier are not included in earlier reports of microRNA profiles in pancreatic cancer tissue. Although the statistical model and the use of 19 microRNAs make the LASSO classifier very robust, this model and the new combinations of two microRNAs need to be validated in a prospective study.

We found a high correlation between the microRNA expression in pancreatic adenocarcinoma and ampullary adenocarcinoma. Only five microRNAs were significantly differentially expressed between these two cancer types. MiR-492, miR-614, miR-198 and miR-196b were on top of the list of differentially expressed microRNAs in both pancreatic and ampullary adenocarcinoma, and these microRNAs were among the most stable in our 19 microRNA diagnostic test.

For validation purposes, we compared our results of microRNA expression in pancreatic adenocarcinomas and controls with those of Bloomston et al ${ }^{20}$ and Szafranska et $a,^{21,26}$ and there was good agreement despite the use of different methods. Five microRNAs (up-regulated: miR-143/143*, miR205, miR-210; down-regulated: miR-148a, miR-375) 
a

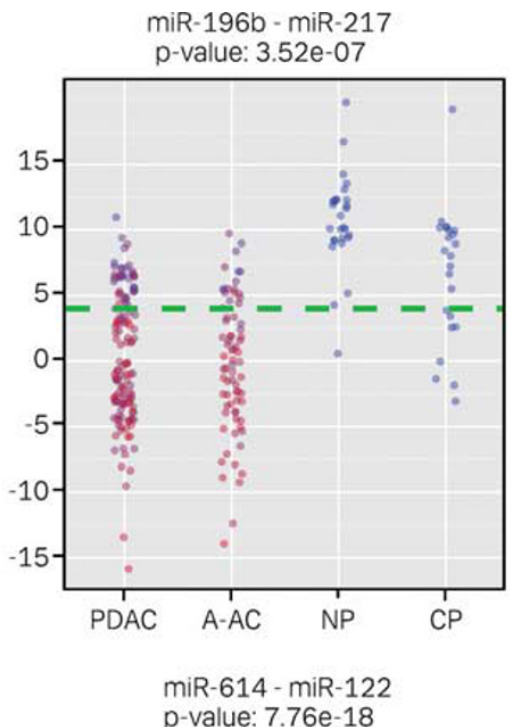

C

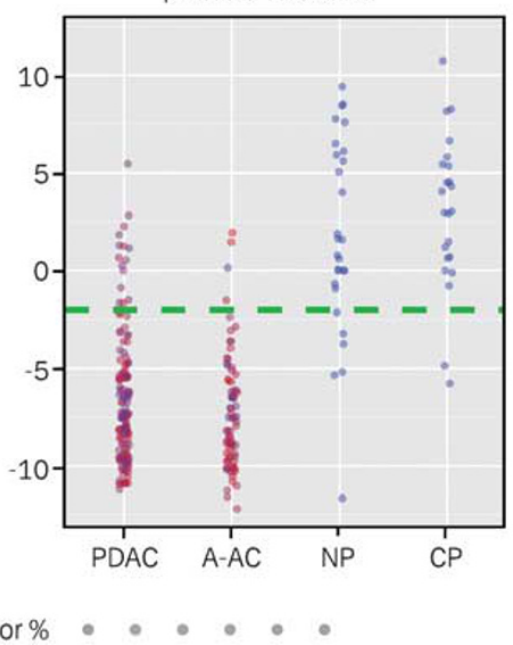

b $\quad m i R-411-m i R-198$

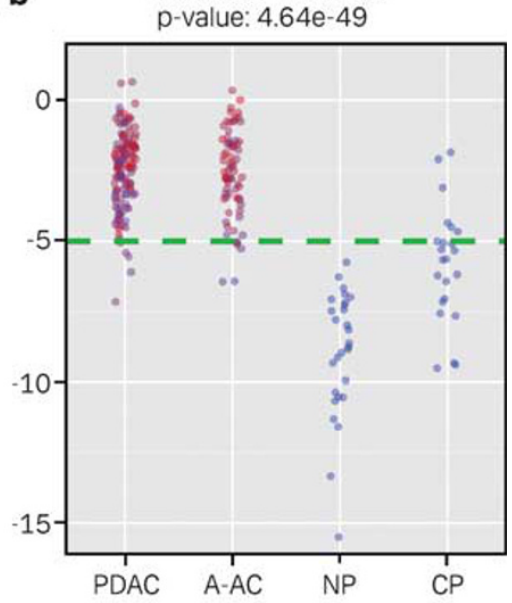

d miR-614-miR-93* p-value: $9.01 \mathrm{e}-18$

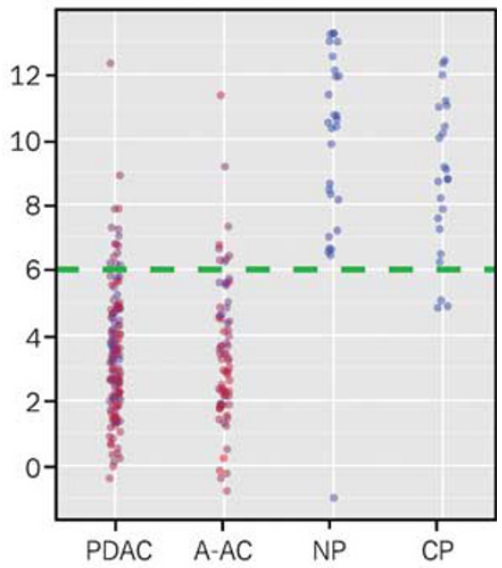

$10080604020 \quad 0$

Figure 3 (a-d) The combinations of two microRNAs given as differences between the microRNAs expressions in the same sample (nonnormalized $C_{\mathrm{t}}$-values). Horizontal lines are showing best cutoff values for separating cancer samples from controls. Color spots showing tumor $\%$ in the tissue samples. The $P$-values given in $(\mathbf{a}, \mathbf{c}, \mathbf{d})$ are for differences in microRNA expression in pancreatic ductal adenocarcinoma (PDAC) and chronic pancreatitis (CP). The $P$-value in (b) is for the differences in microRNA expression differences in PDAC and ampullary adenocarcinoma (A-AC) compared with normal pancreas (NP) and CP.

Table 5 Differences in expressions of two microRNAs as biomarkers to differentiate between pancreatic cancer and controls

\begin{tabular}{lccc} 
MiRNAs & PDAC vS CP & PDAC vs NP & $\begin{array}{c}\text { PDAC }+A-A C \\
\text { VS } C P+N P\end{array}$ \\
\hline mirR-196b-miR-217 & $3.52 \times 10^{-7}$ & $8.59 \times 10^{-20}$ & $4.35 \times 10^{-24}$ \\
mirR-411-miR-198 & $2.63 \times 10^{-13}$ & $5.17 \times 10^{-43}$ & $4.64 \times 10^{-49}$ \\
mirR-614-miR-122 & $7.76 \times 10^{-18}$ & $1.62 \times 10^{-14}$ & $8.64 \times 10^{-38}$ \\
mirR-614-miR-93* & $9.01 \times 10^{-18}$ & $5.56 \times 10^{-24}$ & $2.64 \times 10^{-42}$
\end{tabular}

PDAC, pancreatic ductal adenocarcinoma; A-AC, ampullary adenocarcinoma; CP, chronic pancreatitis; NP, normal pancreas.

were significantly differentially expressed between cancer and normal pancreas both in the present study and earlier studies. ${ }^{20,21,26}$ The studies have all demonstrated that miR-148a was down-regulated in pancreatic adenocarcinomas compared with chronic pancreatitis. This group of microRNAs is therefore well validated for the purpose of separating pancreatic adenocarcinoma, normal pancreas and pancreatitis tissue. Only seven microRNAs described in pancreatic adenocarcinoma by both Bloomston et al ${ }^{20}$ and Szafranka et al ${ }^{21,26}$ could not be validated in our study. However, all these microRNAs (either up- or down-regulated compared with normal pancreas) were also significantly differentially expressed in our study before Bonferonni correction for multiple comparisons, except miR-155 that was not present in our microRNA array. We found several new microRNAs related to pancreatic adenocarcinoma since we used a newly up-dated microRNA array that gives a comprehensive expression profile 
of the total human microRNAs including many newly discovered microRNAs. The studies by Bloomston et $a l^{20}$ and Szafranska et $a l^{21,26}$ are performed with arrays comprehensive for the microRNAs which were described until 2008.

Some of the new microRNAs related to pancreatic adenocarcinoma may have become apparent in our study because we used non-microdissected tumor samples where the stromal tissue can contribute to a distinct microRNA profile. The often prominent desmoplastic stroma in pancreatic ductal adenocarcinomas includes activation of fibroblasts and myofibroblasts transformation, inflammation, neovasculation and enhanced secretion of cytokines, matrix proteins and metalloproteinases. ${ }^{30}$ This stroma plays a role in tumor proliferation, progression, angiogenesis, cell death and matrix remodeling. The observed microRNA expression profiles in each cancer sample may depend on the distribution of tumor cells and stromal tissue. Our study reflects daily clinical practice, where a needle biopsy is used to detect tumor cells in pancreas or metastasis. In the literature no microRNAs are yet described to be related to the development of fibrosis in pancreas.

The knowledge about the role of microRNA in pancreatic cancer and in cancer in general is constantly increasing. However, the role of many recently described microRNAs, like some of them in our study (eg, miR-492 and miR-614), is still undefined. After binding to the RISC microRNAs regulate gene expression posttranscriptionally by a binding of specific mRNA, depending on the target mRNA they can function as oncogene or tumor suppressor. ${ }^{10-19}$ Several microRNAs or miR-families involved in stem cell signaling are identified, for example, miR-21 involved in self-renewal and apoptosis, the let-7 family and miR-200 family involved in pluripotency and cell differentiation. ${ }^{34}$ The miR200 family is down-regulated in cells undergoing epithelial-to-mesenchymal transition (EMT). EMT facilitates tissue remodeling in embryonic development and is an essential early step in tumor metastasizing. ${ }^{35-37}$ Several oncogenic microRNAs are expressed in early stages of development in undifferentiated embryonic cells. However, their expression decreases in differentiated tissue, whereas the opposite holds true for tumor suppressive microRNAs. ${ }^{34}$ Mir-141*, miR-200c* and miR-205 are members of the miR-200 family and we found them all down-regulated in pancreatic adenocarcinomas. MiR-21 was up-regulated in pancreatic adenocarcinomas in this and other studies and has been related to short survival. ${ }^{20,22,38,39}$ Increased miR-21 expression increases the proliferative and invasive potential in several types of cancer, it has anti-apoptotic and pro-angiogenesis properties and its expression may represent an adaptation to a hypoxic environment that favors cancer cell survival. ${ }^{40}$

In accordance with others, we found that miR-148a, miR-216b and miR-217b were some of the miRNAs with the most significantly decreased expression in pancreatic adenocarcinomas compared with chronic pancreatitis and normal pancreas. ${ }^{21,39}$ Over-expression of miR-217 in pancreatic adenocarcinoma cells inhibits tumor cell growth in vivo and in vitro. ${ }^{41}$ Expression of miR-217 is negatively related to KRAS expression. Up-regulation of miR-217 decreases KRAS protein level and reduces the constitutive phosphorylation of AKT in the downstream PI3K-AKT pathway involved in cell growth, differentiation, proliferation and survival. ${ }^{41}$ Down-regulation of miR-148a is an early marker of pancreatic adenocarcinoma and is already decreased in pre-neoplastic PanIN lesions. ${ }^{42}$ At least 27 target genes are known for miR-148a, including bladder cancer associated protein. ${ }^{43}$ In our study, miR-148a expression is related to KRAS mutations in pancreatic and ampullary adenocarcinoma.

Though, many of the microRNAs presented in this study are known to regulate stem cell and cancer cell signaling pathways, each microRNA can potentially regulate nummerous different mRNA in a complex regulatory network. Some of the newly described microRNAs in pancreatic adenocarcinoma have very abnormal expression and their role is still unclear.

We have tried to develop two methods to subclassify ampullary adenocarcinomas into pancreatobiliary or intestinal-type adenocarcinomas by the use of microRNA expression profiles. Both microRNAs classifiers were made with a panel of pancreatic and duodenal adenocarcinomas but without the use of the ampullary cancer samples. Thus, we did not introduce a bias from the histological subclassification in our classifiers. Interesting, 16 $(17 \%)$ samples were classified different than the histologically and immunohistochemically classification by both microRNA classifiers. MicroRNAs are suggested to be new strong cancer classifiers and subclassifiers. ${ }^{44}$

In conclusion, we identified systematic differences in microRNA expression between pancreas tissues, including both cancer cells and stroma, obtained from patients with pancreatic adenocarcinoma and ampullary adenocarcinoma compared with tissue from patients with chronic pancreatitis and normal pancreas. Some of the most differentially expressed microRNAs play a role in normal development, homeostasis or oncogenesis. We identified three new simple combinations of two microRNAs, which roughly discriminate pancreatic and ampullary adenocarcinoma tissue from chronic pancreatitis and normal pancreas. We have then demonstrated that the expression of a panel of 19 microRNAs could separate cancer tissue from non-neoplastic tissue very precisely with sensitivity close to $99 \%$ and an accuracy of $97 \%$. A prospective study with validation of our diagnostic microRNA models in FNAC-biopsies is ongoing. 


\section{Acknowledgements}

We thank Mel Heeran, Krisztina Faludi, Eileen Wong, Herlev Hospital, Copenhagen University Hospital for help with the sectioning of the samples. Dr Anders Molven, Department of Pathology, and Dr Dag Hoem, Department of Surgery, Haukeland University Hospital, Bergen University, Norway are thanked for providing four normal pancreas samples. Mogens Kruhøffer AROS, Aarhus, Denmark is thanked for excellent technical assistance with the miRNA analyses. This study was supported by grants from the Research Council at Herlev Hospital, Augustinus Fonden and 'Læge Sofus Carl Emil Friis og hustru Olga Doris Friis' Legat'. The authors had full access to all the data in the study and had the final responsibility for the decision to submit the manuscript for publication.

\section{Disclosure/conflict of interest}

The authors declare no conflict of interest.

\section{References}

1 Li D, Xie K, Wolff R, et al. Pancreatic cancer. Lancet 2004;363:1049-1057.

2 Siegel R, Naishadham D, Jemal A. Cancer Statistics, 2012. CA Cancer J Clin 2012;62:10-29.

3 Wagner M, Redaelli C, Lietz M, et al. Curative resection is the single most important factor determining outcome in patients with pancreatic adenocarcinoma. Br J Surg 2004;91:586-594.

4 Beger HG, Thorab FC, Liu Z, et al. Pathogenesis and treatment of neoplastic diseases of the papilla of Vater: Kausch-Whipple procedure with lymph node dissection in cancer of the papilla of Vater. J Hepatobiliary Pancreat Surg 2004;11:232-238.

5 Bettschart V, Rahman MQ, Engelken FJ, et al. Presentation, treatment and outcome in patients with ampullary tumours. Br J Surg 2004;91:1600-1607.

6 Albores-Saavedra J, Murakata L, Krueger JE. Noninvasive ad minimally invasive papillary carcinomas of the extrahepatic bileducts. Cancer 2000;89:508-515.

7 Kimura W, Futakawa N, Wada Y, et al. Different clinopathologic findings in two histologic types of carcinoma of papilla of vater. Jpn J Cancer Res 1994;85: 161-165.

8 Zhou H, Schaefer N, Wolff M, et al. Carcinoma of the ampulla of Vater: comparative histologic/immunohistochemical classification and follow-up. Am J Surg Pathol 2004;28:875-882.

9 Sessa F, Furlan D, Zampatti C, et al. Prognostic factors for ampullary adenocarcinomas: tumor stage, tumor histology, tumor location, immunohistochemistry and microsatellite instability. Wirchovs Arch 2007;451: 649-657.

10 Hwang HW, Mendell JT. MicroRNAs in cell proliferation, cell death, and tumorigenesis. Br J Cancer 2006; 94:776-780.

11 Waldman SA, Terzic A. Translating MicroRNA discovery into clinical biomarkers in cancer. JAMA 2007; 297:1923-1925.
12 Li M, Marin-Muller C, Bharadwaj U, et al. MicroRNAs: control and loss of control in human physiology and disease. World J Surg 2009;33:667-684.

13 Nelson KM, Weiss GJ. MicroRNAs and cancer: past, present, and potential future. Mol Cancer Ther 2008;7: 3655-3660.

14 Rachagani S, Kumar S, Batra SK. MicroRNA in pancreatic cancer: pathological, diagnostic and therapeutic implications. Cancer Lett 2010;292:8-16.

15 Farazi TA, Spitzer JI, Morozov P, et al. MiRNAs in human cancer. J Pathol 2010;223:102-115.

$16 \mathrm{Lu} \mathrm{J}$, Getz G, Miska EA, et al. MicroRNA expression profiles classify human cancers. Nature 2005;435: 834-838.

17 Volinia S, Calin GA, Liu CG, et al. MicroRNA expression signature of human solid tumors defines cancer gene targets. Proc Natl Acad Sci USA 2006;103:2257-2261.

18 Landgraf P, Rusu M, Sheridan R, et al. A mammalian microRNA expression atlas based on small RNA library sequencing. Cell 2007;129:1401-1414.

19 Rosenfeld N, Aharonov R, Meiri E, et al. MicroRNAs accurately identify cancer tissue origin. Nat Biotechnol 2008;26:462-469.

20 Bloomston M, Frankel WL, Petrocca F, et al. MicroRNA expression patterns to differentiate pancreatic adenocarcinoma from normal pancreas and chronic pancreatitis. JAMA 2007;297:1901-1908.

21 Szafranska AE, Davison TS, John J, et al. expression alterations are linked to tumorigenesis and nonneoplastic processes in pancreatic ductal adenocarcinoma. Oncogene 2007;26:4442-4452.

22 Dillhoff M, Liu J, Frankel W, et al. MicroRNA-21 is overexpressed in pancreatic cancer and a potential predictor of survival. J Gastrointest Surg 2008;12:2171-2176.

23 Zhang Y, Li M, Wang H, et al. Profiling of 95 microRNAs in pancreatic cancer cell lines and surgical specimens by real-time PCR analysis. World J Surg 2009;33: 698-709.

24 Habbe N, Koorstra JB, Mendell JT, et al. MicroRNA miR-155 is a biomarker of early pancreatic neoplasia. Cancer Biol Ther 2009;8:340-346.

25 Iorio MV, Croce CM. MicroRNAs in cancer: small molecules with a huge impact. J Clin Oncol 2009;27: 5848-5856.

26 Szafranska AE, Doleshal M, Edmunds HS, et al. Analysis of microRNAs in pancreatic fine-needle aspirates can classify benign and malignant tissues. Clin Chem 2008;54:1716-1724.

27 Lee EJ, Gusev Y, Jiang J, et al. Expression profiling identifies microRNA signature in pancreatic cancer. Int J Cancer 2007;120:1046-1054.

$28 \mathrm{Li}$ J, Smyth P, Flavin R, et al. Comparison of miRNA expression patterns using total RNA extracted from matched samples of formalin-fixed paraffin-embedded (FFPE) cells and snap frozen cells. BMC Biotechnol 2007;7:36.

29 Zhang X, Chen J, Radcliffe T, et al. An array-based analysis of microRNA expression comparing matched frozen and formalin-fixed paraffin-embedded human tissue samples. J Mol Diagn 2008;10:513-519.

30 Chu GC, Kimmelman AC, Hezel AF, et al. Stromal biology of pancreatic cancer. J Cell Biochem 2007;101: 887-907.

31 Hamilton SR, Aaltonen LA (editors). World Health Organisation Classification of Tumors. Pathology and Genetics of Tumors of the Gastrointestinal Tract. IARC Press: Lyon, 2000, 219-250. 
32 Mar JC, Kimura Y, Schroder K, et al. Data-driven normalization strategies for high-throughput quantitative RT-PCR. BMC Bioinformatics 2009;10:110.

33 Friedman J, Hastie T, Tibshirani R. Sparse inverse covariance estimation with the graphical lasso. Biostatistics 2008;9:432-441.

34 Navarro A, Monzo M. MicroRNAs in human embryonic and cancer stem cells. Yonsei Med J 2010;51:622-632.

35 Peter ME. Let-7 and miR-200 microRNAs: guardians against pluripotency and cancer progression. Cell Cycle 2009;8:843-852.

36 Gregory PA, Bert AG, Paterson EL, et al. The miR-200 family and miR-205 regulate epithelial to mesenchymal transition by targeting ZEB1 and SIP1. Nat Cell Biol 2008;10:593-601.

37 Thiery JP, Acloque H, Huang RY, et al. Epithelialmesenchymal transitions in development and disease. Cell 2009;139:871-890.

38 Hwang JH, Voortman J, Giovannetti E, et al. Identification of microRNA-21 as a biomarker for chemoresistance and clinical outcome following adjuvant therapy in resectable pancreatic cancer. PLoS One 2010; 14:e10630

39 Nagao Y, Hisaoka M, Matsuyama A, et al. Association of microRNA-21 expression with its targets, PDCD4 and TIMP3, in pancreatic ductal adenocarcinoma. Modern Pathol 2012;25:112-121.

40 Krichevsky AM, Gabriely G. MiR-21: a small multifaceted RNA. J Cell Mol Med 2009;13:39-53.

41 Zhao WG, Yu SN, Lu ZH, et al. The miR-217 microRNA functions as a potential tumor suppressor in pancreatic ductal adenocarcinoma by targeting KRAS. Carcinogenesis 2010;31:1726-1733.

42 Hanoun N, Delpu Y, Suriawinata AA, et al. The silencing of microRNA 148a production by DNA hypermethylation is an early event in pancreatic carcinogenesis. Clin Chem 2010;56:1107-1118.

43 Gress TM, Muller-Pillasch F, Geng M, et al. A pancreatic cancer-specific expression profile. Oncogene 1996;13:1819-1830.

44 Lujambio A, Lowe S. The microcosmos of cancer. Nature 2012;482:347-355.

Supplementary Information accompanies the paper on Modern Pathology website (http://www.nature.com/ modpathol) 